- This study supports the notion that unexplained physical symptoms in dental patients may be psychological in origin and may result from somatization.

- The results suggest that it is important that GDPs exercise caution when providing extensive dental treatment to patients with a record of multiple or recurrent complaints, high attendance and depressive tendencies.

- The findings suggest that when organic pathology is excluded and it is considered that patients' symptoms may be of psychological origin, it is important that the patient is referred to a specialist (eg psychologist, psychiatrist) for psychiatric assessment and appropriate treatment.

\title{
Clinical characteristics of somatization in dental practice
}

\author{
A. De Jongh ${ }^{1}$
}

Objectives This study was a first attempt to derive an estimate of the likely incidence of somatization-specific behaviour in a dental setting and its relationship with both somatic symptoms and symptoms of depression.

Methods Somatization-specific behaviour was operationalized as reporting of inexplicable dental symptoms (eg pain), remarkable frequent attendance at a dental surgery, inexplicably high treatment use or unreasonable requests with regard to treatment.

Results Of the 309 patients surveyed, 8.7\% fulfilled one or more of the criteria for somatization-specific behaviour. This was particularly manifested by a high attendance rate (6.8\%). Women exhibited somatization-specific behaviour significantly more often (73\%) than men (27\%). Support was found for the hypothesis that individuals exhibiting characteristics of somatization-specific behaviour would present themselves to dentists more often with dental complaints and would suffer more from symptoms of depression than patients that did not display these characteristics.

Conclusions The results suggest that somatization is a factor in dental practice.

Dental providers are sometimes confronted with patients that have undergone appropriate dental treatments but who frequently return with ongoing vague symptoms and complaints, such as chronic pain or irritation. While the dental clinician cannot produce a somatic explanation nor give a satisfying treatment of the complaints, the patient requests further investigation and treatment. Typically, fillings are replaced, endodontic treatment is provided or the tooth is extracted but without the forthcoming treatment effects, thereby causing further distress and impairment. ${ }^{1}$

\footnotetext{
1Professor, Department of Social Dentistry and Dental Health Education, Academic Centre for Dentistry Amsterdam, and Centre for Psychotherapy and Psychotrauma, Bilthoven, and Centre for Special Dental Care (SBT), Amsterdam, The Netherlands

${ }^{*}$ Correspondence to: Ad de Jongh, Department of Social Dentistry and Dental Health Education, Academic Centre for Dentistry Amsterdam, Louwesweg 1, 1066 EA Amsterdam, The Netherlands

e-mail:info@psycho-trauma.nl
}

\section{Refereed paper}

Received 27.02.02; Accepted 21.11.02

doi:10.1038/sj.bdj.4810404

๑ British Dental Journal 2003; 195: 151-154
The phenomenon of patients complaining of chronic or recurrent physical symptoms that they themselves attribute to a physical ailment without this being substantiated by medical examination, or laboratory assessment, is generally be referred to as somatization. ${ }^{2-4}$ In its most serious form - somatization disorder - this is a psychiatric syndrome that is defined according to very strict criteria. ${ }^{5}$ To meet the criteria for somatization disorder, the patient should demonstrate a history of many different physical complaints that cannot (or can only partly) be explained by organic pathology or pathophysiological mechanisms, and have caused the patient to take medicine, see a physician, or alter his or her life-style. Prevalence of this diagnosis in women in the United States is estimated to be $0.3 \%$ in the general population, and $0.6 \%$ in those who visit their general practitioner. ${ }^{6,7}$

However, research has shown that only a small number of patients suffer from this psychopathological condition as defined by strict criteria and that somatizers who do not meet full criteria for somatization disorder are much more common. ${ }^{8}$ The Epidemiologic Catchment Area (ECA) study estimated that 4\% of American adults have a lifetime history of multiple unexplained complaints and reported at least one episode during the past year. ${ }^{9}$ Huygen et al. estimate that "chronic nervous functional complaints' play a part in $18 \%$ of consultations with general practitioners. $^{7}$ Thus, it would seem that somatization is a frequently observed phenomenon in primary care practice.

There is a strong presumption that somatization is linked to psychological factors. ${ }^{10}$ For example, it has been argued that somatization should be regarded as a manifestation of psychosocial distress caused by traumatic exposure and radical life changes such as a serious illness, a new job or personal problems. ${ }^{11}$ To this end, somatization is considered to be a "translation' of psychological problems into physical ailments. ${ }^{2}$ Indeed, epidemiological studies show that respondents who can be identified as somatizers have a higher prevalence of psychological problems (particularly mood disorders), and report more frequent use of medical services and higher levels of disability than those who can not. ${ }^{9,12-14}$

There are indications that somatization also plays a role within dentistry. For example, there is evidence to suggest that amalgam-related complaints are an expression of underlying psychological problems. ${ }^{15-16}$ Furthermore, a tendency toward 
somatization is a frequently cited feature of patients diagnosed with temperomandibular disorder, ${ }^{17-18}$ burning mouth syndrome, ${ }^{19}$ and salivary gland complaints. ${ }^{20}$ A more general physical symptom for which an adequate organic pathology cannot always be found is chronic orofacial pain. A study among attenders at a dental pain clinic, mainly referred by their dentist, showed that $91 \%$ of patients with chronic pain could be diagnosed as having a clinically important psychiatric disorder ${ }^{15}$ in accordance to the Diagnostics and Statistical Manual of Mental Disorders (DSM). ${ }^{5}$ The largest single group of this sample consisted of patients with depressive disorders (41\%). The fact that the mean duration of the pain was in excess of 4 years, and that these patients had not been diagnosed as having psychiatric disorders prior to referral to the clinic, suggests that dental clinicians find it difficult to assess the presence of possible underlying psychological factors in orofacial pains. Therefore, it is important that somatizers be identified, not least in the interests of the patient. Recognition of the signs of somatization may prevent them from being subjected to unnecessary and inappropriate treatments, and thus reduce the chance of iatrogenic harm. Moreover, it is not inconceivable that somatization places an extra burden on health services owing to their higher-than-normal treatment use and the costs attached to unnecessary appointments..$^{9,21}$

The present study was a first attempt to derive an estimate of the likely incidence of somatization in general dental practice. Our assumption was that if somatization did occur in dental practice, it would be recognized in individuals who present themselves with vague complaints (eg pain) for which no dental cause can be found, or with unreasonable requests for dental treatment, or with a remarkably high attendance rate (for a variety of complaints or treatments) or with an extremely high treatment use. ${ }^{1,4,23}$ Therefore, somatization was operationalized as the presence of one or more of the previously mentioned clinical characteristics of somatization, which will be further referred to as 'somatization-specific behaviour'.

An additional aim of the current study was to explore the interrelation among somatization specific behaviour, the complaining of somatic symptoms, and symptoms of depression (see Fig. 1). It was predicted that patients with somatization-specific behaviour would present themselves to dentists more often with dental complaints and would show more symptoms of depression.

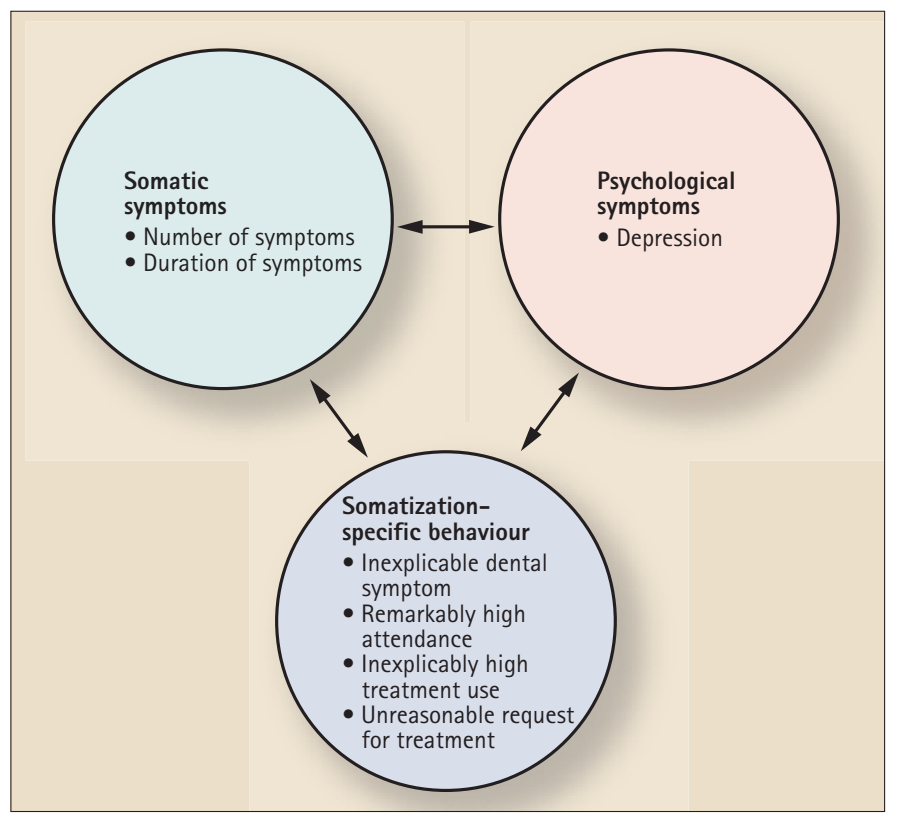

Fig. 1 Relation among the research variables and their operationalizations

\section{MATERIAL AND METHODS \\ Participants}

All dentists working in the city of Amsterdam, whether in private practice or at a dental clinic, were selected for this study. These dentists $(n=93)$ were first approached in writing and then by telephone $(n=82)$. Nineteen dentists ultimately took part in the study. The study was carried out in their practice. Every patient attending the dentist during the recruitment phase (randomly one day per dentist over a period of one month) was screened. The average percentage of patients per dentist was 5.2 (range: 0.6-12.3). Patients were excluded if they were under the age of 16 years or had insufficient command of the Dutch language. Twenty-two patients refused to take part in the study. The final sample consisted of 309 patients. The sex ratio was equal ( $52 \%$ female). The age of the patients varied from 16 to 82 years and averaged 43.6 years ( $\mathrm{SD}=14.9$ ). All sociodemographic groups were represented. An analysis of patients' scores on the depression dimension of the multidimensional psychopathology indicator, SCL-90, led to the conclusion that they did not differ from the normative mean score for the general Dutch population. ${ }^{24}$

\section{Methods}

Patients were recruited consecutively on the day of their appointment with the dentist in the waiting room. Participants were handed the questionnaires and given standardized verbal instructions on how to fill these in. After treatment or consultation, the dentist completed a checklist consisting of five items that indexed somatization-specific behaviour. For each patient the presence of one or more of the following characteristics was recorded:

\section{An inexplicable dental symptom}

2. A remarkably high attendance (for different symptoms or treatments)

3. An inexplicably high treatment use

4. An unreasonable request with regard to treatment, or

5. None of the above characteristics.

There were checkboxes for each characteristic. More than one characteristic could be ticked. The presence of one or more of these characteristics was considered as somatization-specific behaviour in the dental setting. Prior to the study each dentist was given a detailed instruction on how to use the checklist.

\section{Measures}

A patient questionnaire constructed for this study contained general questions (including questions on gender, age and education) and one item that asked whether the patient had suffered from one or more dental or oral symptoms (pain, irritation etc.) in the previous month. Patients were asked to rate their responses on a 3-point scale, with 1 indicating 'no symptoms', 2 indicating 'one symptom' and 3 'more than one symptom'. Patients who indicated to have suffered one symptom or more were asked to indicate whether they were bothered by it either for days/weeks (shortstanding) or for months/years (long-standing).

The depression scale of the Revised Symptom Checklist, SCL-90-R $\mathrm{R}^{24,25}$ was used to assess symptoms of depression. This scale consists of 16 items that reflect a representative range of the manifestations of clinical depression. Symptoms of dysphoric mood and effect are represented as are signs of withdrawal of life interest, lack of motivation and loss of vital energy. In addition, feelings of hopelessness, thoughts of suicide, and other cognitive and somatic correlates of depression are included (eg feelings of isolation, and general listlessness; 16 items). Patients are requested to indicate on a 5-point scale the 
amount of complaints they experienced during the previous week ( $1=$ none; $5=$ very many). The responses are summed (score range: 16-80). Cronbach's alpha of the depression scale in the present study was 0.96).

\section{Statistical analysis}

The average scores of two groups were compared by means of Student's $t$-tests for independent groups. Comparisons between more groups were carried out using one-way ANOVAs, and chi-square tests to determine the distribution of proportions between groups. For all statistical analyses a p-value of less than 0.05 was considered statistically significant.

\section{RESULTS}

Characteristics of somatization-specific behaviour were observed in $8.7 \%$ of the patients studied. Somatization-specific behaviour was particularly manifested by high attendance (6.8\%). Table 1 gives a breakdown of the various forms of somatization-specific behaviour.

\begin{tabular}{|c|c|c|}
\hline & $\begin{array}{c}\text { Number } \\
\text { of patients }\end{array}$ & $\begin{array}{l}\text { Percentage } \\
5 \quad(\%)\end{array}$ \\
\hline Inexplicably high treatment use & 1 & 0.3 \\
\hline Inexplicable dental symptom & 3 & 1.0 \\
\hline Unreasonable request with regard to treatment & 6 & 1.9 \\
\hline Remarkably high attendance & 21 & 6.8 \\
\hline Patient displays none of the above characteristics & 282 & 91.3 \\
\hline
\end{tabular}

The dentists indicated that women (73\%) exhibited one of the above characteristics more often than men $(27 \%)$ did $\left(\chi^{2}(1)=4.3\right.$; $P<0.05)$. Somatization-specific behaviour appeared to be independent of age.

There was a significant difference in the proportions of patients who indicated that they suffer from dental or oral symptoms between those who fulfilled the criteria of somatization-specific behaviour and those who did not $\left(\chi^{2}(2)=7.8 ; P<0.005\right)$. Approximately two-thirds of the patients that exhibited somatization-specific behaviour (68\%) indicated that they suffer from dental or oral symptoms, while one-third (32\%) did not experience any symptoms. Patients exhibiting somatization-specific behaviour appeared to have significantly higher $(t(252)=2.9 ; P<0.05)$ scores on the SCL-90 subscale 'depression' $(\mathrm{M}=30.9$; $\mathrm{SD}=14.7)$ than those not exhibiting this behaviour $(\mathrm{M}=21.4$; $\mathrm{SD}=7.2)$.

Forty-one per cent of all patients complained of pain or other symptoms in the previous month. Within the latter group, 28\% of patients reported one symptom and 13\% more than one. Of all patients complaining, 55.1\% indicated that their symptoms had begun a relatively short time ago (29.9\% several days ago, 25.2\% several weeks ago), while $44.9 \%$ reported that they had suffered for much longer (29.9\% several months ago, 15.0\% several years ago).

Table 2 shows that patients complaining of multiple dental symptoms exhibited somatization-specific behaviour more often than those with one symptom or no symptoms at all $\left(\chi^{2}(2)=9.0\right.$, $P<0.05)$. A similar pattern can be established with regard to the duration of these symptoms $\left(\chi^{2}(2)=8.4 ; P<0.05\right)$. Table 2 also shows that patients who complained of multiple symptoms were more prone to depression than those with one or no symptoms $(\mathrm{F}(2.242)=5.6 ; P<0.005)$.

Patients with long-standing symptoms exhibited relatively more somatization-specific behaviour than did patients without symptoms or with symptoms of short duration. There was no relationship between symptom duration and signs of depression.
Table 2 The extent to which patients suffered somatic (ie dental) symptoms in the previous month in relation to somatization-specific behaviour and symptoms of depression

\begin{tabular}{lccc}
\hline $\begin{array}{l}\text { Number of } \\
\text { symptoms }\end{array}$ & $\begin{array}{c}\text { Patients } \\
n(\%)\end{array}$ & $\begin{array}{c}\text { Somatization- } \\
\text { specific behaviour (\%) }\end{array}$ & $\begin{array}{c}\text { Depression* } \\
\text { M (SD) (\%) }\end{array}$ \\
\hline No symptoms & $155(59)$ & 5 & $21.5(8.1)^{\mathrm{a}}$ \\
One symptom & $71(28)$ & 11 & $21.5(6.7)^{\mathrm{a}}$ \\
Multiple symptoms & $35(13)$ & 20 & $26.8(11.5)^{\mathrm{b}}$ \\
\hline${ }^{*}$ a and b are used to indicate mutual differences between groups: $b>\mathrm{a}(P<0.05)$
\end{tabular}

\section{DISCUSSION}

The results of this study show that almost 9\% of the participating patients exhibited one or more characteristics of somatization according to our operationalization. This was particularly manifested by a remarkably high attendance rate. With regard to the relationship between somatization and gender it was found that women exhibited somatization-specific behaviour almost three times as often as men. The results supported our hypothesis that individuals exhibiting characteristics of somatization-specific behaviour would present themselves to dentists more often with dental complaints and would suffer more from symptoms of depression than those who did not display such characteristics.

There proved to be a significant relationship between somatization-specific behaviour and the complaining of physical symptoms. Patients who indicated that they suffer from multiple or long-standing symptoms were particularly likely to exhibit somatization-specific behaviour. For instance, somatizationspecific behaviour was found four times more frequently among patients experiencing multiple symptoms than it was in the group with no symptoms. The finding that women exhibited somatization-specific behaviour more often than men may be explained by a cultural factor; that is, that in our society it is probably a stronger taboo for men to complain or show weakness.

Patients with multiple symptoms also scored higher for depression than people with no symptoms. This finding is supported by other observers of a relationship between depression and somatic symptoms. ${ }^{5,11,13}$ On the one hand, this may be because the symptoms have such an effect on patients' mood and so impair their performance of day-to-day tasks that they consequently take a bleak view of life. On the other hand, the patients' feelings of depression may make them more inclined to interpret physical sensations, including pain, as having an impairing effect. This suggests that taking a purely dental approach to an inexplicable dental symptom will not automatically relieve the problem. To this end, it has been advocated that psychiatric involvement is essential to the assessment and management of patients showing unexplained physical symptoms, particularly those who admit to having consulted multiple healthcare professionals in the past. ${ }^{26}$

In seeking to generalize the results, this study has several limitations. The study targeted dental practices in Amsterdam. Moreover, the response rate was low and, given that more than $20 \%$ of the respondents thought that their command of the Dutch language was insufficient for them to fill in the questionnaire, the behaviour of patients from ethnic minorities may not be studied in sufficient depth. However, although there are concerns over generalizability, the importance of this study is that the findings are in accordance with the results of previous studies on somatization, showing that consultation frequency - inherently related to somatization - was related to gender, somatic complaints and depression. ${ }^{13,21}$ To this end, the findings provide support for the validity of the operationalization of somatization used in the present study. 
In conclusion, the results of this preliminary study support the notion that somatization is a factor in dental practice. It is to be hoped that more information on the role and the importance of this phenomenon within the context of dentistry will become available in future years. Until it does, it is to be recommended that clinicians exercise caution when providing extensive dental treatment to patients with a record of long-standing multiple or recurrent complaints, high attendance and depressive tendencies. This is in order to prevent overtreatment, and thus the risk of iatrogenic harm.

The support and assistance from Dideric Lock and Martine de Groot are gratefully acknowledged and appreciated.

1. Jongh $A$, de. Oppassen voor valkuilen: de problematiek van de somatiserende patiënt [Beware of pitfalls: Somatization in dental practice.] Ned Tijdsch Tandheelkd 1997; 104: 159-151.

2. Lipowski Z J. Somatization: The concept and its clinical application. Am J Psychiatry 1988; 145: 1358-1368.

3. Fink P. Somatization - beyond symptom count. J Psychosom Res 1996; 40: 7-10.

4. Portegijs P J M, Horst van der F G, Proot I M, Kraan H F, Gunther N C H F, Knottnerus, J A. Somatization in frequent attenders of general practice. Soc Psychiatry Psychiatr Epid 1996; 31: 29-37.

5. American Psychiatric Association. Diagnostic and statistical manual of mental disorders (4th ed). Washington, DC: American Psychiatric Association, 1994.

6. Robins L N, Helzer J E, Weisman M M et al. Lifetime prevalence of specific psychiatric disorders in three sites. Arch Gen Psychiatry 1984; 41: 949-958.

7. de Gruy F, Columbia L, Dickinson P. Somatization disorder in a family practice. J Fam Practice 1987; 25: 45-51.

8. Huygen F J A, Hoogen $H J$ M van den, Logt A T van de, Smits A J A. Nerveusfunctionele klachten in de huisartsenpraktijk. I. Een epidemiologisch onderzoek [Nervous-functional complaints in general practice I: An epidemiological study]. Ned Tijdschr Geneeskd 1984; 128: 1321-1327.

9. Robins L N, Rogier D A. Psychiatric disorders in America. New York: The Free Press; 1991.

10. Bridges K W, Goldberg D P. Somatic presentation of DSM-III psychiatric disorders in primary care. J Psychosom Res 1985; 29: 563-569.
11. Lloyd G G. Psychiatric syndromes with a somatic presentation. J Psychosom Res 1986 30: 113-120.

12. Barsky A J. A comprehensive approach to the chronically somatizing patient. JPsychosom Res 1998; 45: 301-306.

13. Escobar JI, Rubio M, Canino G, Karno M. Somatic Symptom Index (SSI): A new and abridged somatization construct. Prevalence and epidemiological correlates in two large community samples. J Nerv Ment Dis 1989; 177: 140-146.

14. Howland R H. General health, health care utilization, and medical comorbidity in dysthymia. J Psych Med 1993; 23: 211-238.

15. Kupfer G B, Traenckner, I, Ganss, C, Gieler U. Psychological, allergic, and toxicological aspects of patients with amalgam-related complaints. Psychother Psychosom 2002; 71:223-232.

16. Malt U F, Nerdrum P, Oppedal B, Gundersen R, Holte M, Lone J. Physical and mental problems attributed to dental amalgam fillings: A descriptive study of 99 self-referred patients compared with 272 controls. Psychosom Med 1997; 59: 32-41.

17. Pakhurst C L. Controversies in the aetiology of temporomandibular disorders. Part 1. Temporomandibular disorders: All in the mind? Prim Dent Care 1997; 4: 25-30.

18. Van der Laan G J, Duinkerke A S, Luteijn F, van de Poel A C. Role of psychologic and social variables in TMJ pain dysfunction syndrome (PDS) symptoms. Community Dent Oral Epidemio/1988; 16: 274-277.

19. Jerlang B B. Burning mouth syndrome (BMS) and the concept of alexithymia. A preliminary study. J Oral Pathol Med 1997; 26: 249-253.

20. Votta T J, Mandel L. Somatoform salivary complaints. Case reports. NYState Dent J 2002; 68: 22-26.

21. Hughes A, Hunter S, Still D, Lamey P J. Psychiatric disorders in a dental clinic. Br DentJ 1989; 166: 16 .

22. Escobar J I, Golding J N, Hough R L, Karno M, Burnam M A, Wells K B. Somatization in the community: Relationship to disability and use of services. Am J Public Health 1987; 77: 837-840.

23. Escobar $\mathrm{J}$ I. Developing practical indexes of somatization for use in primary care. JPsychosom Res 1997; 4: 323-328.

24. Arrindell W A, Ettema J H M. SCL-90: Handleiding bij een multidimensionele psychopathologie-indicator [SCL-90: Manual for a multidimensional psychopathology-indicator.] Lisse: Swets \& Zeitlinger, 1986.

25. Derogatis L R. Administration, scoring and procedures manual I for the R(evised) version and other instruments of the psychopathology rating scale series. Baltimore, MD: Clinical Psychometrics Research Unit, John Hopkins University School of Medicine, 1977.

26. Coulthard P, Morris S, Hamilton A J. Unexplained physical symptoms in dental patients. BrDent J 1998; 184: 378-382. 